\title{
(6) OPEN ACCESS \\ Detection of gluten immunogenic peptides in the urine of patients with coeliac disease reveals transgressions in the gluten-free diet and incomplete mucosal healing
}

\author{
María de Lourdes Moreno, ${ }^{1}$ Ángel Cebolla, ${ }^{2}$ Alba Muñoz-Suano, ${ }^{2}$ \\ Carolina Carrillo-Carrion, ${ }^{2}$ Isabel Comino, ${ }^{1}$ Ángeles Pizarro, ${ }^{3}$ Francisco León, ${ }^{4}$ \\ Alfonso Rodríguez-Herrera, ${ }^{5}$ Carolina Sousa ${ }^{1}$
}

\begin{abstract}
${ }^{1}$ Facultad de Farmacia, Departamento de

Microbiología y Parasitología, Universidad de Sevilla, Sevilla, Spain

${ }^{2}$ Biomedal S.L., Sevilla, Spain ${ }^{3}$ Unidad Clínica de Aparato Digestivo, Hospital

Universitario Virgen del Rocío, Sevilla, Spain

${ }^{4}$ Celimmune, Bethesda,

Maryland, USA

${ }^{5}$ Unidad de Gastroenterología y

Nutrición, Instituto Hispalense de Pediatría, Sevilla, Spain
\end{abstract}

Correspondence to Professor Carolina Sousa, Facultad de Farmacia, Departamento de Microbiología y Parasitología, Universidad de Sevilla,

C/ Profesor García González 2. Sevilla 41012, Spain;

csoumar@us.es

Received 10 June 2015 Revised 23 September 2015 Accepted 25 September 2015 Published Online First 25 November 2015

CrossMark

To cite: Moreno ML, Cebolla Á, Muñoz-Suano A, et al. Gut 2017;66:250257.

\section{ABSTRACT}

Objective Gluten-free diet (GFD) is the only management for coeliac disease (CD). Available methods to assess GFD compliance are insufficiently sensitive to detect occasional dietary transgressions that may cause gut mucosal damage. We aimed to develop a method to determine gluten intake and monitor GFD compliance in patients with $C D$ and to evaluate its correlation with mucosal damage.

Design Urine samples of 76 healthy subjects and 58 patients with $C D$ subjected to different gluten dietary conditions were collected. A lateral flow test (LFT) with the highly sensitive and specific G12 monoclonal antibody for the most dominant gluten immunogenic peptides (GIP) and a LFT reader were used to quantify GIP in solid-phase extracted urines.

Results GIP were detectable in concentrated urines from healthy individuals previously subjected to GFD as early as 4-6 h after single gluten intake, and remained detectable for 1-2 days. The urine assay revealed infringement of the GFD in about $50 \%$ of the patients. Analysis of duodenal biopsies revealed that most of patients with CD $(89 \%)$ with no villous atrophy had no detectable GIP in urine, while all patients with quantifiable GIP in urine showed incomplete intestinal mucosa recovery.

Conclusion GIP are detected in urine after gluten consumption, enabling a new and non-invasive method to monitor GFD compliance and transgressions. The method was sensitive, specific and simple enough to be convenient for clinical monitoring of patients with $C D$ as well as for basic and clinical research applications including drug development.

Trial registration number NCT02344758.

\section{INTRODUCTION}

The mainstay of the management of coeliac disease (CD) is a strict life-long compliance with glutenfree diet (GFD). ${ }^{1}{ }^{2}$ However, full compliance with the GFD is very difficult to achieve given the ubiquity of gluten as a common food additive, as well as due to dietary habits, high costs of the GFD and the social restrictions it imposed on patients. ${ }^{3}$ At least one-third of patients with CD do not fully

\section{Significance of this study}

What is already known on this subject?

- Ensuring the compliance of gluten-free diet (GFD) by patients with coeliac disease (CD) is a crucial step in the management of CD. According to several reports, dietary transgressions are relatively frequent (36\% to $55 \%$ ).

- Available methods to assess GFD compliance are insufficiently sensitive to detect occasional dietary transgressions that may cause gut mucosal damage. In addition, these tests only measure the consequences of dietary transgressions. There is a need for accurate, reliable and non-invasive tools to help avoid the harmful consequences of dietary indiscretions in CD.

- Certain gluten immunogenic peptides (GIP) are resistant to gastrointestinal digestion and can interact with the immune system of patients with $C D$ to trigger an autoimmune response against transglutaminase and other antigens. A proportional fraction of the GIP absorbed in the gastrointestinal tract makes it to the circulation and is excreted in urine.

\section{What are the new findings?}

- In this study, we describe for the first time a non-invasive, novel, specific and reliable approach to detect and monitor the presence of GIP in urine. We have been able to distinguish gluten consumers from non-consumers by a quantitative lateral flow test using anti- $\alpha$-gliadin monoclonal antibodies.

- GIP were sensitively detected in human urine samples in positive correlation with the amount of gluten intake. Our results indicate that the ingestion of amount as little as $>25 \mathrm{mg}$ of gluten in processed bread (lower limit of the amount that appears detrimental for most patients with $C D$ ) can be detected in urine. 


\section{Significance of this study}

-We confirmed the poor correlation of serological tests with mucosa healing as well as the shortcomings of diet questionnaires to assess GFD compliance. In contrast, a good correlation between the presence of GIP in urine and higher damage in the intestinal epithelium (Marsh II/III score) was demonstrated.

\section{How might it impact on clinical practice in the foreseeable future?}

- The incorporation of this simple immunological assay in urine could overcome some key unresolved scientific and clinical problems in CD monitoring. We identify four main applications: (1) detection of inadvertent lapses after appearance of acute symptoms, (2) non-compliance of the GFD before any anatomic damage, (3) monitoring the adherence to the treatment in initial period after diagnosis when patients are less familiar with the diet and (4) the accurate diagnosis and management of diet in non-responsive and true refractory CD (RCD) cases.

- This technique may also serve to assess the efficacy of new experimental drugs or strategies to eliminate GIP during digestion in patients with CD.

- It is a significant advance that opens up new possibilities for the gastroenterologist to control the treatment of this disease, assess GFD compliance and improve the quality of life of patients with $C D$.

adhere to a GFD. ${ }^{4}$ In addition, $36 \%$ to $55 \%$ of patients who declare to fully adhere to a GFD do not achieve histological remission, probably because of inadvertent lapses in daily gluten intake. $^{25-7}$

There is no consensus regarding the optimal frequency of monitoring the GFD or the best tools for assessing compliance. ${ }^{8}$ Despite the availability of diverse GFD adherence markers, they have significant limitations and are insufficiently sensitive to detect occasional transgressions that may impede full gut mucosa recovery. ${ }^{9-17}$ Determination of gluten peptides in faeces has recently been proposed for direct verification of GFD compliance. ${ }^{18}$ Urine has unique advantages for sampling such as lower cost, non-invasiveness, easy collection, transport, storage and relative homogeneity versus faeces. ${ }^{19-21}$

In this study, we have overcome technical challenges and have shown the feasibility of measuring gluten immunogenic peptides (GIP) in urine. These peptides are resistant to gastrointestinal digestion and account for most of the immunotoxic reactions in $\mathrm{T}$ cells of patients with $\mathrm{CD}$. We assessed compliance with the GFD in healthy and coeliac individuals by estimation of GIP in urine with anti-GIP immunochromatographic strips (IC strips). Notably, the presence of GIP in the urine of patients with CD correlated with intestinal atrophy. The method appears to be sensitive, specific and simple, and should be useful for clinical monitoring of the GFD in patients with CD and to support efforts in pharmaceutical coeliac research.

\section{MATERIAL AND METHODS Study patients}

A total of 134 subjects ( 86 women and 48 men) were randomly enrolled, of which 58 were patients with CD (age range 3-64 years) and 76 healthy subjects (age range 3-57 years). A total of 69 adults ( $>16$ years of age) and 65 children were included.

Exclusion criteria for all study patients included the presence of known medical disease, use of prescription medications and antibiotics in the 2 months prior to the inclusion in the study. Moreover, healthy patients had no digestive disease symptoms or family history of $\mathrm{CD}$.

The local ethics committee of the Hospital Virgen de Valme (Sevilla, Spain) approved the study protocol. Written consent was obtained from adult patients and, in the case of children, from parents or legal guardians.

\section{Duodenal mucosa evaluation}

In this work, at least four endoscopic biopsies of the distal duodenum and two biopsies from the duodenal bulb were processed. Duodenal biopsies were fixed in $10 \%$ buffered formalin embedded in paraffin and sectioned at 4-5 $\mu \mathrm{m}$ thickness. The haematoxylin-eosin stained sections were assessed. The study and quantification of intraepithelial lymphocytes (IEL) were performed by immunohistochemistry using automated platform Leica BOND-III. The proportion and distribution of the IEL along the glands were determined in all the biopsies. The mucosal specimens were graded independently according to the Marsh-Oberhuber's classification. ${ }^{22}{ }^{23}$ Biopsies were interpreted by expert gastrointestinal pathologists (blinded to the clinical data). We used the cut-off of $\geq 40$ IEL/100 enterocytes for the Marsh classification.

\section{Serology}

Serum IgA anti-tissue transglutaminase (TTG) and IgA anti-gliadin antibodies (AGA) were measured using commercial ELISA (InmunoCAP Phadia, Uppsala, Sweden). Titres of $\leq 10 \mathrm{U} / \mathrm{mL}$ were considered negative and those of $>10 \mathrm{U} / \mathrm{mL}$ were considered positive.

\section{Urine sampling}

All participants were provided sterile containers. Mid-stream urine samples $(50-100 \mathrm{~mL})$ from volunteers were collected and stored at $-20^{\circ} \mathrm{C}$ until analysis. Urine specimens were mixed by gentle inversions for at least 30 min before processing.

Urine samples from healthy subjects and patients with $C D$ were collected under different gluten dietary conditions and times as follows: (1) to differentiate gluten consumers from non-consumers, random specimen urines from healthy individuals $(\mathrm{n}=10)$ while following a non-standardised glutencontaining diet (GCD) and healthy controls on GFD $(n=10)$; (2) to test the appearance and elimination of ingested gluten in urine, all excreted urines from healthy subjects $(n=13)$ for 4 days: the first 3 days on a GFD and the fourth and last day on a GCD; (3) to check the detection limit of urinary gluten detection, all excreted urines from healthy subjects on a GFD $(n=4)$ after administration of controlled gluten microdoses (25 and $50 \mathrm{mg}$ ); (4) to test the sensitivity of gluten measurement in $24 \mathrm{~h}$ total urine, $24 \mathrm{~h}$ pooled urine samples from six healthy controls on GCD and one coeliac patient on GFD and (5) to follow-up of GFD compliance, random urines from 76 healthy volunteers (consuming a GCD) and 58 patients with CD on GFD for $>2$ years.

\section{Competitive ELISA}

A commercial competitive ELISA kit based on G12 monoclonal antibody (Biomedal, S.L., Spain) was used to analyse the gluten content of the fragments of wheat white bread administered to the volunteers in the controlled gluten challenge. 


\section{Food inquiries}

All healthy subjects were instructed to follow specific dietary restrictions. GCD was ensured by the ingestion of at least a portion of pasta, bread or whole grain of cereals like wheat, barley and rye per day. Adults with CD recorded a collection of all foods ingested on the 4 days prior to urine sampling. At the end of the study, compliance with dietary conditions was ascertained through a structured interview.

The dietary history was carefully reviewed by a dietician. 'Risk products' were defined as products known to possibly contain gluten, but for which the exact amount of gluten could not be calculated due to missing brand information.

\section{Urine peptides concentration}

Urine samples were concentrated and cleaned-up using solidphase extraction (SPE) technique. SampliQ C18 cartridges $(500 \mathrm{mg}, 6 \mathrm{~mL})$ supplied by Agilent (Wilmington, Delaware, USA) were preconditioned following manufacturer's recommendations. Separately, a $5 \mathrm{~mL}$ mixture of $50 \%$ urine in trifluoroacetic acid was centrifuged $10 \mathrm{~min}$ at $2500 \mathrm{~g}$. The resultant supernatant was applied to the cartridge and the target compounds were eluted with $1 \mathrm{~mL}$ of phosphate-buffered saline for further use in IC assays.

\section{IC test for detection of GIP}

The G12 IC assay was modified from the guidelines of the manufacturer (GlutenTox Stick, Biomedal, Sevilla, Spain). After the SPE of the sample, $100 \mu \mathrm{L}$ of the blind concentrated were added into a well (multiwell plate). Then, a G12 IC-strip was dipped into the well for $30 \mathrm{~min}$ and allowed to air-dry afterwards. The IC strip was introduced into the cassette of a lateral flow test (LFT) reader and was irradiated with light and the reflection measured.

\section{LFT quantitation}

To establish a correlation between GIP content and output signal of the IC strips, urine from individuals with CD without gluten peptides was used as control. Individuals with CD were selected based on its histology and serology. To verify negative gluten intake of the patients, we conducted a qualitative analysis by IC strips in concentrated urines as well as in faeces, according to the protocol established by Comino et al. ${ }^{18}$ Different concentrations of gliadin standard were spiked to control urine in concentrations ranging from 6.25 to $1000 \mathrm{ng} / \mathrm{mL}$.

GlutenTox Reader (Biomedal, Seville, Spain) was used as reader for GIP quantification in IC strips. Test sticks were scanned by an optical detector, which continually recorded measurement data.

\section{Statistical analysis}

All results are expressed as mean SD. Each urine sample was analysed in triplicate. Positive control and buffer blanks were included in each assay. Statistical analyses were performed with GraphPad Prism 6 for Windows. Unpaired, two-tailed Student's $\mathrm{t}$ test was applied and $\mathrm{p}<0.05$ was considered as statistically significant.

We used the non-parametric Fisher's exact test, CochranArmitage test and Spearman's correlation coefficient to assess the presence of GIP in urines of adults with CD and the severity of Marsh lesion and the estimates of the level of association between two variables. We used SAS (V.9.4;Cary, North Carolina, USA) for all statistical calculations. All $\mathrm{p}$ values presented are two-sided.

\section{RESULTS}

\section{Detection of GIP in urine}

We attempted to determine whether gluten peptides are excreted and could be detected in urine. Initially, samples were collected from non-coeliac subjects $(n=10)$ following a GCD, and IC strips were dipped into samples. No visible signals in sticks were found in spite of gluten consumption. As gluten peptides were expected to be at very low concentrations in urine, we decided to perform a SPE to improve the feasibility of GIP detection. Urines from 20 healthy adults were concentrated and divided in two groups, one group $(n=10)$ received a nonstandardised diet in which gluten was consumed daily, and other group $(n=10)$ was subjected to GFD for a week. We tested the presence of GIP in urines from the individuals included on the two different diet groups. GIP were detected in all concentrated urines of subjects on GCD. However, we detected no GIP in any of the concentrated urines of the volunteers on GFD. These results strongly indicated that the signal was dependent on gluten intake.

\section{Calibration of GlutenTox Reader to quantify the output signal of urine IC strips}

To correlate the GIP concentration and the output signal of urine IC strips, the LFT analyser GlutenTox Reader was calibrated with five series of gliadin standards. The mean value at each standard was calculated, as well as its SD and the relative SD. The calibration function, which fits to a Rodbard function (data not shown) were calculated and then introduced in the anti-GIP LFT reader software to quantify the GIP in urines. The quantification limit (QL) of the technique was established as $6.25 \mathrm{ng} \mathrm{GIP} / \mathrm{mL}$ urine. The uncertainty was $17.6 \%(5.5-7.35 \mathrm{ng}$ $\mathrm{GIP} / \mathrm{mL}$ urine). The limit of technique detection (LDT), defined as the minimum intensity that the reader is able to detect was below the lower standard of quantification (LDT $=42.7-$ $48.37 \mathrm{mV})$.

\section{In vivo GIP monitoring of healthy individuals after consumption of various gluten-controlled diets}

To examine the chronology of appearance and elimination of ingested gluten in urine, a total of 13 healthy volunteers were subjected to different diet conditions and the collected urines were analysed by their GIP content by using anti-GIP LFT. We collected all excreted urines for 1 day in unrestricted conditions (GCD) from healthy individuals. Then, individuals were submitted to a GFD for 3 days and all excreted urines were collected until measurable GIP content became undetectable. Subsequently, a GCD was reintroduced and urines were also collected. Between three and six different urines per day were collected. Figure $1 \mathrm{~A}$ shows an example of representative analysed IC strips from one healthy subject (aH6). Kinetics of GIP excretion from four healthy volunteers $(\mathrm{aH} 1, \mathrm{aH} 5, \mathrm{aH} 8$ and aH12) revealed that GIP content was undetectable after 16-34 h from the beginning of the GFD in all tested individuals (figure 1B). GIP were detected in urines after 3-9 h of the reintroduction of dietary gluten. In 12 of 13 healthy individuals on GFD, the amounts of GIP in urine were below the QL of the method. The subjects had a good compliance with GFD; however, a peak of $40 \mathrm{ng} \mathrm{GIP} / \mathrm{mL}$ urine was detected on the third day of the trial as shown in the aH12 graph. When interviewed at the end of the study, that volunteer confirmed consumption of yogurt with cereals (including wheat) few hours before the urine collection. 


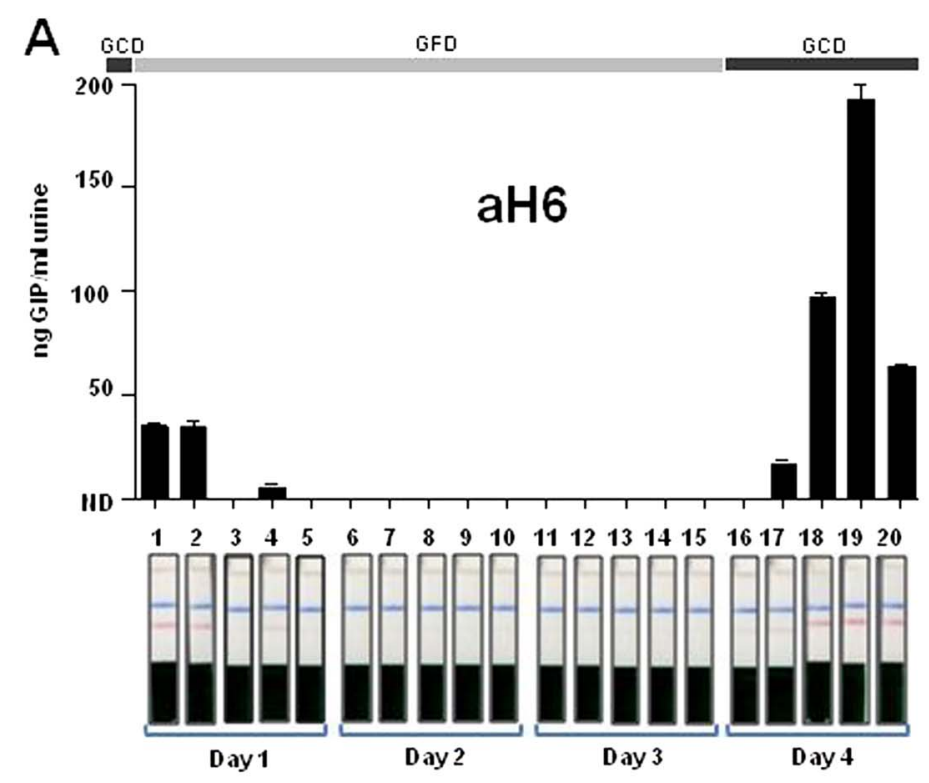

B
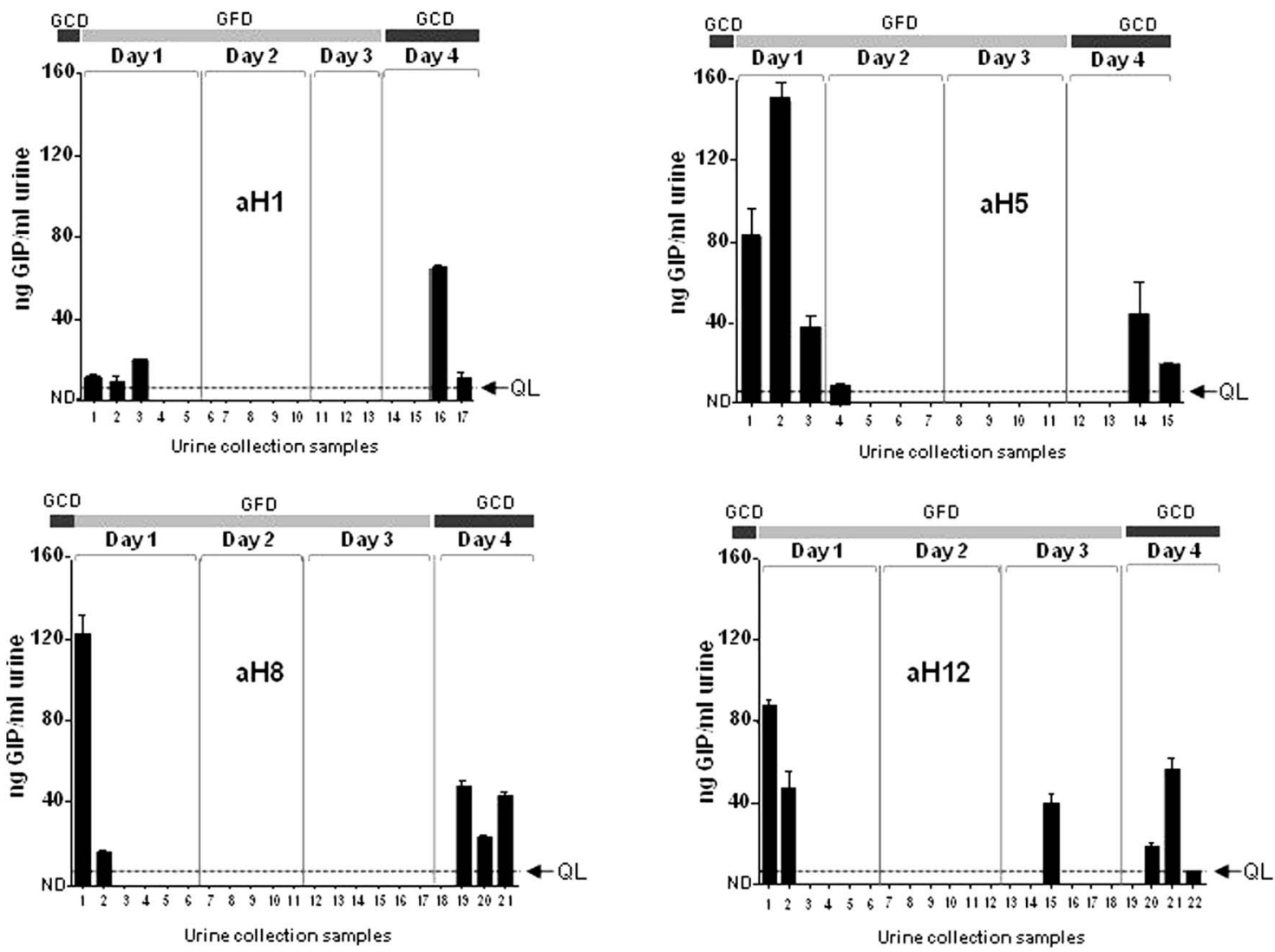

Figure 1 Determination of the time to elimination and to appearance of GIP in urines of healthy individuals. Urine samples from healthy individuals, regularly consuming gluten, who were subject to a GFD were collected until reactive peptides became undetectable. Three to six different urine samples per day were collected for 4 days. (A) One representative example of the gluten excretion kinetics from the trial with the representative immunochromatographic strip example of the trial was performed with the samples collected during the study period of one subject. Blue stripes represent an internal positive control that indicates that the stick worked properly; pink stripes indicate the presence of gluten. (B) Kinetics of gluten-derived peptides excreted from four healthy volunteers. GIP, gluten immunogenic peptides; GCD, gluten-containing diet; GFD, gluten-free diet; QL, quantification limit; ND, not detectable.

To test whether the anti-GIP LFT was capable of detection in urine, the minimum gluten consumption known to cause histological abnormalities, two doses of gluten $(25$ and $50 \mathrm{mg})$ were administered to four healthy subjects (figure 2). One single type of gluten was used in all cases through a standardised piece of white bread containing $25 \mathrm{mg}$ gluten. An initial microdose of 

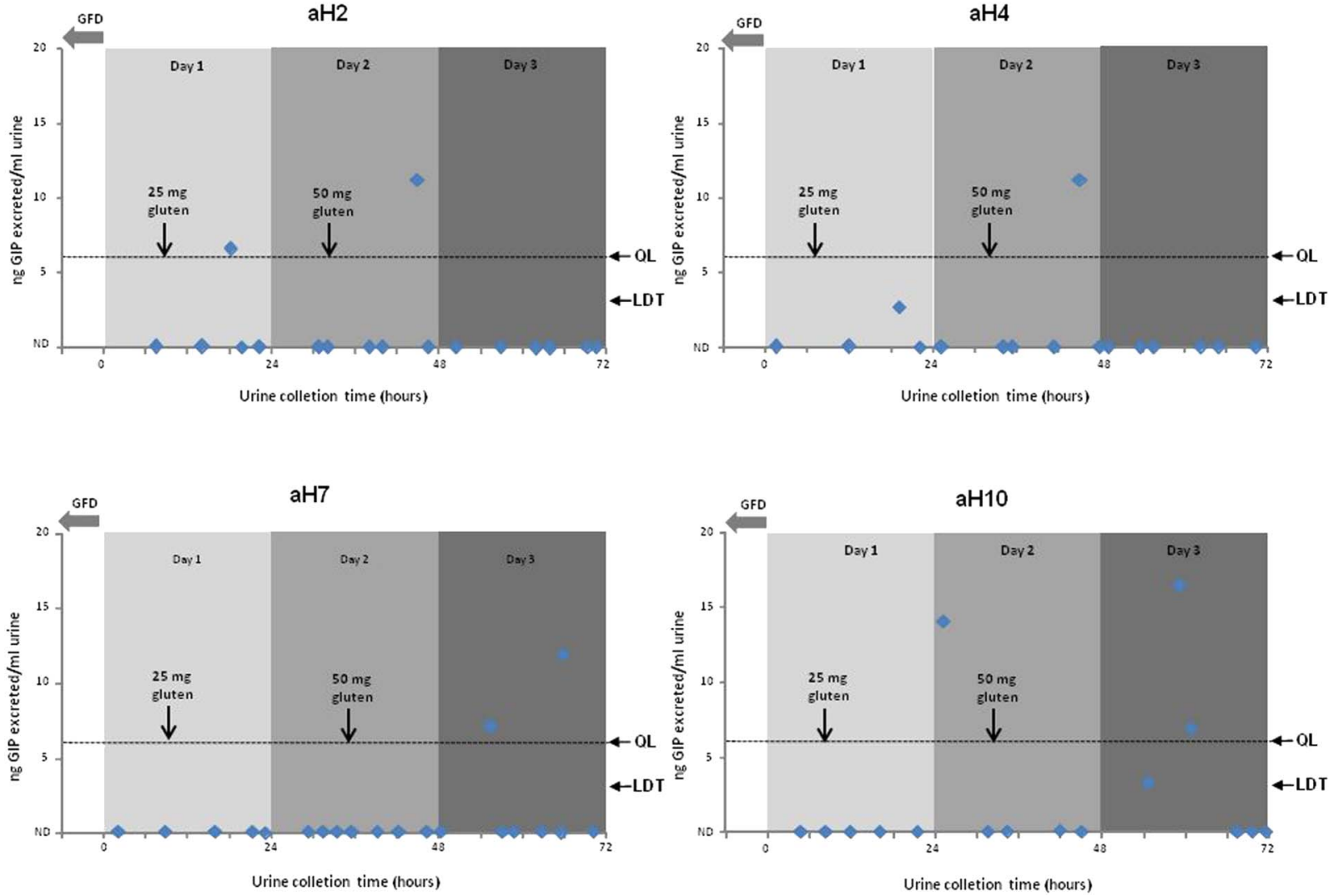

Figure 2 In vivo monitoring of urinary excretion of gluten peptides in healthy individuals on a GFD with consumption of controlled gluten challenges. Two doses of gluten were administered $(25$ and $50 \mathrm{mg})$ to four subjects. Four independent experiments with samples were run in triplicate. GFD, gluten-free diet; GIP, gluten immunogenic peptides; QL, quantification limit; LDT, limit of technique detection; ND, not detectable.

$25 \mathrm{mg}$ gluten was administered and the GIP content was measured in urine by using anti-GIP LFT. Then, doses of $50 \mathrm{mg}$ were given and GIP measurements were repeated. GIP became detectable in urine in all analysed individuals at the $50 \mathrm{mg}$ dose. Administration of $25 \mathrm{mg}$ gluten resulted in enough GIP in urine to be visibly detected (over LDT) in three out of four individuals $(\mathrm{aH} 2, \mathrm{aH} 4$ and $\mathrm{aH} 10)$ although only in $\mathrm{aH} 2$ and $\mathrm{aH} 10$ the signal was quantifiable (over QL). The signal of aH10 was clearly over the QL. Therefore, the limit of detection (LD) of this method could be established as $>25 \mathrm{mg}$ of ingested gluten.

The sensitivity of the method in random single urines was similar to the 24-h urines (data not shown). The measurement of gluten peptides by collecting 24-h total urine may increase the chances of detection of ingestion of low amounts of gluten.

\section{Follow-up of GFD compliance in patients with CD with the anti-GIP lateral flow urine test}

The high percentage of patients with CD with insufficient gut mucosal healing is primarily attributed to inadvertent dietary lapses and minor voluntary transgressions. Therefore, there is a need for an accurate marker that would allow short-term monitoring of GFD compliance by physicians and patients. To assess whether the proposed method is suitable for monitoring gluten ingestion in patients with $\mathrm{CD}$, a study was conducted to measure GIP in urine of 76 healthy volunteers (42 adults and 34 children) who were consuming a GCD, and 58 patients with CD (27 adults and 31 children) on long-term GFD (figure 3). After consumption of a GCD, all healthy subjects showed gluten excretion in urine. The range of GIP in urine of healthy subjects ranged from 6.54 to $604 \mathrm{ng} \mathrm{GIP} / \mathrm{mL}$ (in adults) and from 6.54 to $369 \mathrm{ng} \mathrm{GIP} / \mathrm{mL}$ (in children).
The presence of GIP in urine of patients with CD on GFD revealed the existence of a high percentage of non-compliance of the GFD. GIP in urine were detectable in $48 \%$ of adults and $45 \%$ of children. However, GIP content in urine was below the QL of the method in 70\% of adults and 71\% of children with CD. In the remainder individuals with $\mathrm{CD}$, GIP content ranged from 9.27 to $78.12 \mathrm{ng} \mathrm{GIP} / \mathrm{mL}$ and from 9.33 to $29.78 \mathrm{ng} \mathrm{GIP} / \mathrm{mL}$ urine (in adults and children, respectively).

\section{Dietary transgressions in adult patients with CD: correlation with mucosal damage}

To establish a correlation between GIP in urine of adults with $\mathrm{CD}$ and the occurrence of mucosal damage, we conducted a histological study of 25 intestinal biopsies of adults with CD who had followed a GFD for at least 2 years (figure 4).

Only 13 out of the 25 patients were GIP- (52\%) and none of them had histological architectural damage in the mucosa (ie, all were Marsh 0-I with no Marsh II/III). Of the 13 of the GIPindividuals, 5 had Marsh 0 (normal mucosa) and 8 had Marsh I (elevated IEL without architectural changes). All the GIPpatients were TTG/AGA - as well. Conversely, six out of seven adults with CD having clear histological abnormalities (Marsh II-crypt hyperplasia and Marsh III-mucosal atrophy) had detectable level of GIP in urine (86\%), and only one subject with Marsh III had detectable but not quantifiable amount of GIP in urine.

Fisher's exact test showed a significant relation $(p=0.0007)$ between the presence of quantifiable GIP (>QL) and severity of Marsh scale. The strong association was observed to be statistically significant (Cochran-Armitage Trend Test $p=0.0005$ ). Moreover, GIP values showed a significant correlation with the 
$p<0,0001$

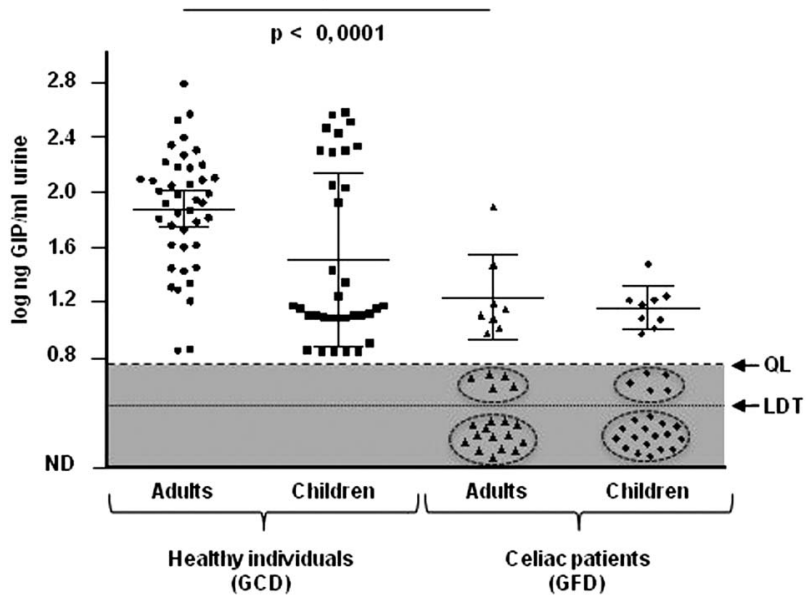

Figure 3 Detection and quantification of GIP in urine samples of healthy and individuals with CD. Patients with $C D(n=58)$ were on GFD and healthy individuals $(n=76)$ on GCD. Each point represents the mean absorbance value of one urine sample from individuals at optical density of $450 \mathrm{~nm}$. According to the QL of technique, individuals with a higher or equal GIP value than QL were considered positive (GIP++) for the presence of GIP, while those with lower GIP content but higher than LDT were considered positive not quantifiable (GIP+) and those with lower GIP content than LDT were considered negative (GIP-); ${ }^{*} \mathrm{p}<0.0001$ (unpaired, two-tailed Student's $t$ test). $C D$, coeliac disease; GIP, gluten immunogenic peptides; GFD, gluten-free diet; GCD, gluten containing diet; QL, quantification limit; LDT, limit of technique detection; ND, not detectable.

severity of intestinal mucosa damage (Spearman's correlation, $\mathrm{r}=0.75$ ). As additional supportive investigation, dietary histories from adults with CD were reviewed. We found that all responders reported full GFD compliance. Some patients were suspected to have consumed gluten in meals prepared away from

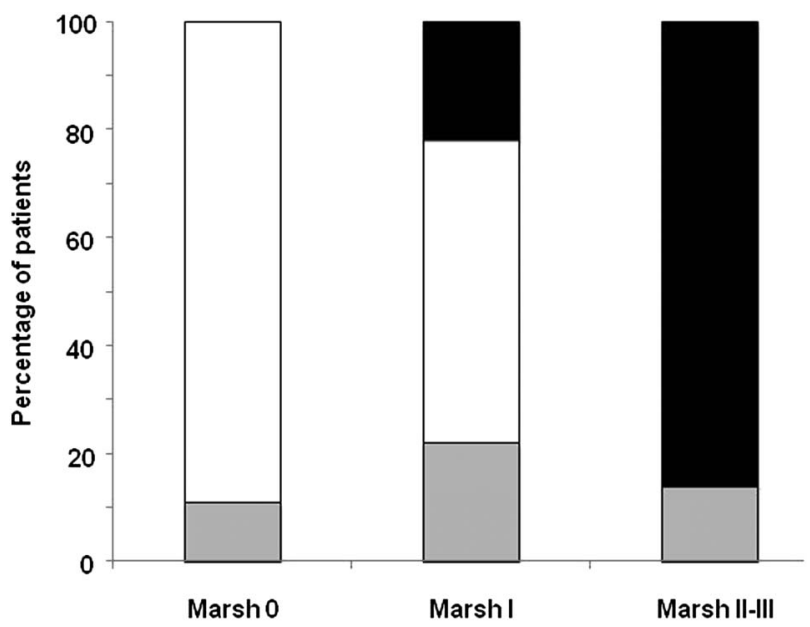

Figure 4 Correlation between the presence of GIP in urine and small bowel histology in adult patients with $\mathrm{CD}$. Histological appearance determined by the Marsh scale of the severity of mucosal lesion (Marsh I-III). GIP - (white bar), absence of GIP in urine; GIP+ (grey bar), visual presence of GIP not quantifiable in urine ( $>$ LDT $<$ QL); GIP ++ (black bar), presence of GIP visible and quantifiable in urine ( $>Q L)$. $p=0.0007$ (Fisher's exact test). Values are expressed as percentage of patients. CD, coeliac disease; GIP, gluten immunogenic peptides; LDT, limit of technique detection; $\mathrm{QL}$, quantification limit. home (eg, Spanish tomato soup, sausages and potato chips). Because at least one-third of adults with CD showed GIP in urine $>$ QL, it could be concluded that the collection of dietary intake did not reflect the gluten intake, whether voluntary or involuntary. Moreover, no correlation was found between dietary history and mucosal healing.

Unlike the excellent correlation of GIP with histology, serological data had no correlation with mucosal damage. Positivity in anti-TTG and/or AGA was found in only four individuals, two with Marsh I and the other two with Marsh II. Five patients with severe lesion in mucosa were not positive in either TTG or AGA (71.4\%).

Hence, this study indicated that only urine GIP detection correlated with compliance with the GFD and with mucosal healing in patients with CD on a GFD.

\section{DISCUSSION}

In this study, we describe for the first time a novel, specific and reliable approach to detect and monitor the presence of GIP in urine by means of G12 IC strips. The recovery of measurable amounts of gluten peptides in urine indicates that gluten has been absorbed by the intestinal mucosa, has reached the circulation and has been filtered by the kidney.

To date, available methods to monitor GFD compliance only measure the consequences of dietary transgressions and many studies have reported poor effectiveness. ${ }^{24-31}$ The use of endoscopies to collect biopsies and assess mucosal healing is the gold standard; however, it is invasive, expensive and not a practical method for serial monitoring. Hence, there is a need for accurate, non-invasive tools to help avoid the harmful consequences of dietary indiscretions in CD.

The resistance of gluten in gastrointestinal digestion ensures that a significant fraction of the ingested gluten is excreted. A previous report described a new methodology for GFD monitoring through the determination of 33 -mer equivalent peptidic epitopes content in faeces. ${ }^{18}$ However, gluten-containing food also contains other gluten peptides that present immunogenicity and they could also be excreted. Methodology based on the G12 antibody demonstrated its ability to recognise content of other peptides immunogenic in patients with CD. ${ }^{32}{ }^{33}$ Given the greater convenience of urine over stool collection in some settings, the herein proposed urine gluten test would become another useful monitoring tool in clinical practice for follow-up of the GFD compliance.

IC strips are standard clinical assays in urine in many diseases. The use of IC strips coupled with an IC reader in urine of patients with $\mathrm{CD}$ could provide a quantitative measurement of dietary infringement, providing significant advantages in the follow-up of GFD compliance. In this study, we have been able to distinguish gluten consumers from non-consumers by using G12 dipsticks in urine. A wide range of gluten peptide amounts (from 6.54 to $604 \mathrm{ng}$ ) could be detected in urine after GCD. The time of gluten peptide excretion in urine was proved to be between 1 and 2 days after gluten ingestion, in contrast to 3-4 days in stools. ${ }^{18}$ Interindividual diversity (weight, sex, age, gut microbiota, etc), type of gluten-containing food (beer, pasta, bread, cookies, etc), the daily amount of liquid intake and the accompanying diet may have a considerable impact on the resultant GIP concentration and excretion time in urine.

The concept of 'daily gluten tolerable intake' has received special attention and daily gluten consumption $>10-50 \mathrm{mg}$ appears detrimental for many patients with CD. ${ }^{24} 34-36$ Our results indicate that the gluten ingestion of $>25 \mathrm{mg}$ in processed bread could be detected in urine. 
Since dietary transgressions are relatively frequent in patients with $\mathrm{CD},{ }^{4537}$ a method to determine inadvertent lapses or voluntary gluten intake is a long-overdue necessity to help patients with CD manage their lives and avoid the harmful effects of gluten exposure while on a GFD. In the current study, we demonstrated the presence of GIP in patients with CD who had supposedly consumed long-term GFD (45\% and 48\% in children and adults, respectively). These results were consistent with reports showing that $\sim 30 \%-50 \%$ of patients with CD on GFD remain mucosal atrophy. More importantly, our results indicate that there is a correlation between the absence of GIP in urine and healing of the gut intestinal epithelium. In addition, the histological analysis revealed that $100 \%$ of the adults with CD with higher damage in the epithelia (Marsh II/III) had consumed gluten according to the detection of GIP in urine. In contrast, our data confirmed the poor correlation of serological tests with mucosal healing, consistent with other studies, ${ }^{12-14}$ as well as the shortcomings of the dietary history questionnaires to assess GFD compliance.

Refractory CD (RCD) is defined by persistent symptoms and signs of malabsorption despite gluten exclusion for $>12$ months with ongoing intestinal villous atrophy. ${ }^{38-43}$ As there is no effective method to determine whether refractory symptoms are due to hypersensitivity to trace amounts of dietary gluten or to inadvertent gluten exposure, the methodology proposed in this work might be a useful way to better diagnose and manage RCD cases.

In this article, we have described how to monitor gluten intake by a simple immunological assay in urine, thereby overcoming some key unresolved scientific and clinical problems in CD monitoring. We identify four main applications: (1) the monitoring of short-term and long-term GFD compliance, (2) assessment of the efficacy of experimental non-dietary treatments in patients with CD, (3) detection of inadvertent lapses of daily gluten ingestion and (4) differential diagnosis of RCD from dietary non-compliance.

Future studies with larger number of patients and samples will further validate the clinical relevance and the applications of the detection of recent GFD transgressions in urine for the optimal management of CD.

Acknowledgements The authors are grateful to Professor Garry Courtney, consultant gastroenterologist at St Luke's Hospital, Kilkenny (Ireland), for his valuable suggestions in the writing of the manuscript. They are grateful to the generous volunteer subjects who enrolled in the study. They also thank Verónica Segura and Manuel Moreno for their assistance with the assays.

Contributors Study concept and design: MLM, ÁC and CS; acquisition of data: MLM, CC-C and AM-S; data analysis and interpretation: MLM, ÁC, AM-S, ÁP, FL, AR-H and CS; technical and material support: MLM, CC-C, IC, AM-S, APP and AR-H manuscript drafting: MLM, ÁC, FL and CS; critical revision of the manuscript: $A C, F L$ and CS.

Funding This work was supported by grants from Ministerio de Ciencia e Innovación and FEDER funds (DELIAC, IPT-2011-0952-900000), Asociación de Celíacos de Madrid y Sensibles al Gluten and Corporación Tecnológica de Andalucía (SINGLUCHECK, 1737/0118).

Competing interests $A C$ and FL own stock in Biomedal SL. Other authors have declared no conflict of interest. The method of this manuscript was included in a patent application by MLM, CS, AR-H and ÁC as inventors with the assigned number P201400569.

Patient consent Obtained.

Ethics approval The local Ethics Committee of the Hospital Virgen de Valme (Sevilla, Spain).

Provenance and peer review Not commissioned; externally peer reviewed.

Open Access This is an Open Access article distributed in accordance with the Creative Commons Attribution Non Commercial (CC BY-NC 4.0) license, which permits others to distribute, remix, adapt, build upon this work non-commercially, and license their derivative works on different terms, provided the original work is properly cited and the use is non-commercial. See: http://creativecommons.org/ licenses/by-nc/4.0/

\section{REFERENCES}

1 Bernardo D, Peña AS. Developing strategies to improve the quality of life of patients with gluten intolerance in patients with and without coeliac disease. Eur J Intern Med 2012:23:6-8.

2 Hall NJ, Rubin GP, Charnock A. Intentional and inadvertent non-adherence in adult coeliac disease. A cross-sectional survey. Appetite 2013;68:56-62.

3 Freeman HJ. Non-dietary forms of treatment for adult celiac disease. World $\mathrm{J}$ Gastrointest Pharmacol Ther 2013;4:108-12.

4 Barratt SM, Leeds JS, Sanders DS. Quality of life in coeliac disease is determined by perceived degree of difficulty adhering to a gluten-free diet, not the level of dietary adherence ultimately achieved. J Gastrointest Liver Dis 2011;20:241-5.

5 Matoori S, Fuhrmann G, Leroux JC. Celiac disease: a challenging disease for pharmaceutical scientists. Pharm Res 2013;30:619-26.

6 Tio M, Cox MR, Eslick GD. Meta-analysis: coeliac disease and the risk of all-cause mortality, any malignancy and lymphoid malignancy. Aliment Pharmacol Ther 2012:35:540-51.

7 Stoven S, Murray JA, Marietta E. Celiac disease: advances in treatment via gluten modification. Clin Gastroenterol Hepatol 2012;10:859-62.

8 Bai J, Zeballos E, Fried M, et al. WGOOMGE practice guideline coeliac disease. World Gastroenterol News 2012;2:S1-8.

9 Dipper CR, Maitra $S$, Thomas R, et al. Anti-tissue transglutaminase antibodies in the follow-up of adult coeliac disease. Aliment Pharmacol Ther 2009;30: 236-44.

10 Vives-Pi M, Takasawa S, Pujol-Autonell I, et al. Biomarkers for diagnosis and monitoring of celiac disease. J Clin Gastroenterol 2013:47:308-13.

11 Vallejo-Diez S, Bernardo D, Moreno ML, et al. Detection of specific $\lg A$ antibodies against a novel deamidated 8-Mer gliadin peptide in blood plasma samples from celiac patients. PLOS ONE 2013;8:e80982.

12 Sharkey LM, Corbett G, Currie E, et al. Optimising delivery of care in coeliac disease - comparison of the benefits of repeat biopsy and serological follow-up. Aliment Pharmacol Ther 2013:38:1278-91.

13 Tursi A, Brandimarte G, Giorgetti GM. Lack of usefulness of anti-transglutaminase antibodies in assessing histologic recovery after gluten-free diet in celiac disease. J Clin Gastroenterol 2003:37:387-91.

14 Rashtak S, Ettore MW, Homburger HA, et al. Comparative usefulness of deamidated gliadin antibodies in the diagnosis of celiac disease. Clin Gastroenterol Hepatol 2008;6:426-32

15 Kaukinen K, Sulkanen S, Mäki M, et al. IgA-class transglutaminase antibodies in evaluating the efficacy of gluten-free diet in coeliac disease. Eur J Gastroenterol Hepatol 2002;14:311-15.

16 Kaukinen $\mathrm{K}$, Peräaho $\mathrm{M}$, Lindfors $\mathrm{K}$, et al. Persistent small bowel mucosal villous atrophy without symptoms in coeliac disease. Aliment Pharmacol Ther 2007;25:1237-45.

17 Tursi A, Brandimarte G, Giorgetti GM, et al. Endoscopic and histological findings in the duodenum of adults with celiac disease before and after changing to a gluten-free diet: a 2-year prospective study. Endoscopy 2006;38:702-7.

18 Comino I, Real A, Vivas S, et al. Monitoring of gluten-free diet compliance in celiac patients by assessment of gliadin 33-mer equivalent epitopes in feces. Am J Clin Nutr 2012;95:670-7.

19 Esteban M, Castaño A. Non-invasive matrices in human biomonitoring: a review. Environ Int 2009:35:438-49.

20 Pinches $\mathrm{M}$, Betts $\mathrm{C}$, Bickerton $\mathrm{S}$, et al. Evaluation of novel renal biomarkers with a cisplatin model of kidney injury: gender and dosage differences. Toxicol Pathol 2012:40:522-33.

21 Li PKT, Burdmann EA, Mehta RL. Acute kidney injury: global health alert. Transplantation 2013:5:653-7.

22 Marsh MN. Gluten, major histocompatibility complex, and the small intestine: a molecular and immunobiologic approach to the spectrum of gluten sensitivity ('celiac sprue'). Gastroenterology 1992;102:330-54.

23 Oberhuber G, Granditsch G, Vogelsang H. The histopathology of coeliac disease: time for a standardized report scheme for pathologists. Eur J Gastroenterol Hepatol 1999:11:1185-94.

24 Herman ML, Rubio-Tapia A, Lahr BD, et al. Patients with celiac disease are not followed up adequately. Clin Gastroenterol Hepatol 2012;10:893-9.

25 Tack GJ, Verbeek WH, Schreurs MW, et al. The spectrum of celiac disease: epidemiology, clinical aspects and treatment. Nat Rev Gastroenterol Hepatol 2010;7:204-13

26 Walker MM, Murray JA. An update in the diagnosis of coeliac disease. Histopathology 2011;59:166-79.

27 Duerksen DR, Wilhelm-Boyles C, Parny DM. Intestinal permeability in long-term follow-up of patients with celiac disease on a gluten-free diet. Dig Dis Sci 2005:50:785-90. 
28 Ertekin V, Selimoğlu MA, Turgut A, et al. Fecal calprotectin concentration in celiac disease. J Clin Gastroenterol 2010;44:544-6.

29 Duerksen DR, Wilhelm-Boyles C, Veitch R, et al. A comparison of antibody testing, permeability testing, and zonulin levels with small-bowel biopsy in celiac disease patients on a gluten-free diet. Dig Dis Sci 2010;55:1026-31.

30 Balamtekın N, Baysoy G, Uslu N, et al. Fecal calprotectin concentration is increased in children with celiac disease: relation with histopathological findings. Turk J Gastroenterol 2012:23:503-8.

31 Capone P, Rispo A, Imperatore N, et al. Fecal calprotectin in coeliac disease. World J Gastroenterol 2014;20:611-12

32 Morón B, Bethune MT, Comino I, et al. Toward the assessment of food toxicity for celiac patients: characterization of monoclonal antibodies to a main immunogenic gluten peptide. PLOS ONE 2008:3:e2294.

33 Comino I, Real A, Moreno ML, et al. Immunological determination of gliadin 33-mer equivalent peptides in beers as specific and practical analytical methods to assess safety for celiac patients. J Sci Food Agric 2013;93:933-43.

34 Catassi C, Fabiani E, lacono G, et al. A prospective, double blind, placebo controlled trial to establish a safe gluten threshold for patients with celiac disease. Am J Clin Nutr 2007:85:160-6.

35 Akobeng AK, Thomas AG. Systematic review: tolerable amount of gluten for people with coeliac disease. Aliment Pharmacol Ther 2008:27:1044-52.
36 Gibert A, Kruizinga AG, Neuhold $S$, et al. Might gluten traces in wheat substitutes pose a risk in patients with celiac disease? A population-based probabilistic approach to risk estimation. Am J Clin Nutr 2013;97:109-16.

37 Silvester JA, Rashid M. Long-term follow-up of individuals with celiac disease: an evaluation of current practice guidelines. Can I Gastroenterol 2007;21: $557-64$.

38 Woodward J. The management of refractory coeliac disease. Ther Adv Chronic Dis 2013:4:77-90.

39 Rubio-Tapia A, Hill ID, Kelly CP, et al. ACG clinical guidelines: diagnosis and management of celiac disease. Am J Gastroenterol 2013;108:656-76.

40 Arguelles-Grande C, Brar P, Green PH, et al. Immunohistochemical and T-cell receptor gene rearrangement analyses as predictors of morbidity and mortality in refractory celiac disease. J Clin Gastroenterol 2013;47:593-601.

41 Ilus T, Kaukinen K, Virta LJ, et al. Refractory coeliac disease in a country with a high prevalence of clinically-diagnosed coeliac disease. Aliment Pharmacol Ther 2014;39:418-25

42 Rubio-Tapia A, Murray JA. Classification and management of refractory coeliac disease. Gut 2010;59:547-57.

43 Nijeboer P, van Wanrooij RL, Tack GJ, et al. Update on the diagnosis and management of refractory coeliac disease. Gastroenterol Res Pract 2013:2013:518483 